\title{
Fibrosarcoma paratesticular: una neoplasia maligna muy infrecuente
}

\author{
Blasco Alfonso JE, Sales Maicas MA*, Pallás Costa Y.
}

Servicio de Urología. *Servicio de Anatomía Patológica. Hospital General de Requena. Valencia.

Actas Urol Esp. 2006;30(7):707-710

\section{RESUMEN}

FIBROSARCOMA PARATESTICULAR: UNA NEOPLASIA MALIGNA MUY INFRECUENTE

Presentamos los hallazgos clínicos y patológicos de un sarcoma paratesticular de cordón espermático muy infrecuente. El tumor fue un fibrosarcoma en un varón de 55 años de edad que debutó de forma atípica con un crecimiento rápido. Se practicó orquiectomía radical con ligadura inguinal de pedículo sin terapia postoperatoria. Tras 5 años de seguimiento no hay signos clínicos ni radiológicos de recidiva locorregional ni a distancia. Concluimos que la cirugía radical es la única alternativa terapéutica, aún cuando presente crecimiento rápido, siendo en nuestro caso el pronóstico favorable.

Palabras clave: Fibrosarcoma. Neoplasia paratesticular. Cordón espermático.

\section{ABSTRACT}

PARATESTICULAR FIBROSARCOMA. A RARE MALIGN NEOPLASM

Hereby we wish to present the clinical and pathological results of a rare paratesticular sarcome of the spermatic cord. This tumor was a fibrosarcoma in a 55 year old male that started in a most unusual way and developed very quickly. We practised a radical orquitectomy with inguinal ligation of the pedicule without post-operatory therapy. After a 5 year follow up, there is no trace of clinical or radiological recurrence in the region or metastasis. We consider radical surgery to be the only therapeutic alternative, even for cases of fast developing, being in our case a good pronostic.

Key words: Fibrosarcoma. Paratesticular neoplasm. Spermatic cord.

$\mathrm{L}$ os tumores paratesticulares (TP) ya sean de la rete testis del epidídimo o del cordón espermático se presentan clínicamente como masas que afectan a la región inguinal o escrotal. Se trata, en general, de una patología poco frecuente cuya incidencia es muy difícil de estimar dado que la mayor parte de las publicaciones se limitan a series cortas y casos aislados ${ }^{1}$.

La neoplasia maligna que nos ocupa se enmarca dentro del grupo de los tumores del cordón espermático. Dichas neoplasias tienen el mismo espectro morfológico que los tumores de los tejidos blandos, siendo más frecuentes los de estirpe benigna.

La compleja histogénesis de los TP está muy relacionada con el desarrollo embriológico de dichas estructuras ${ }^{2}$ aunque los sarcomas del cordón se cree que derivan de las células mesenquimatosas.

Presentamos un caso de fibrosarcoma de cordón espermático por su rareza y presentación atípica ya que existen muy pocos casos comunicados en la literatura mundial.

\section{CASO CLINICO}

Varón de 55 años de edad con antecedentes de colecistectomía y sin antecedentes urológicos de interés.

Es remitido a la Consulta Externa de Urología por aumento de tamaño hemiescrotal izquierdo de 6 meses de evolución que ha presentado un crecimiento rápido en los últimos 60 días. No refiere dolor ni clínica miccional. 
La exploración física puso de manifiesto la presencia de una masa mamelonada que no transiluminaba, de consistencia firme, dependiente de cordón no adherida a planos y de unos $6 \mathrm{~cm}$ de diámetro máximo. Ausencia de adenopatías en cadenas inguinales y supraclaviculares. Los marcadores testiculares (alfa fetoproteína, beta-HCG y LDH) resultaron negativos.

El estudio ecográfico escrotal confirmó la presencia de una masa sólida de señal hiperecogénica heterogénea de $5,4 \times 3,5 \mathrm{~cm}$ sin aparentes áreas de necrosis que desplazaba medialmente al testículo izquierdo (Fig. 1).

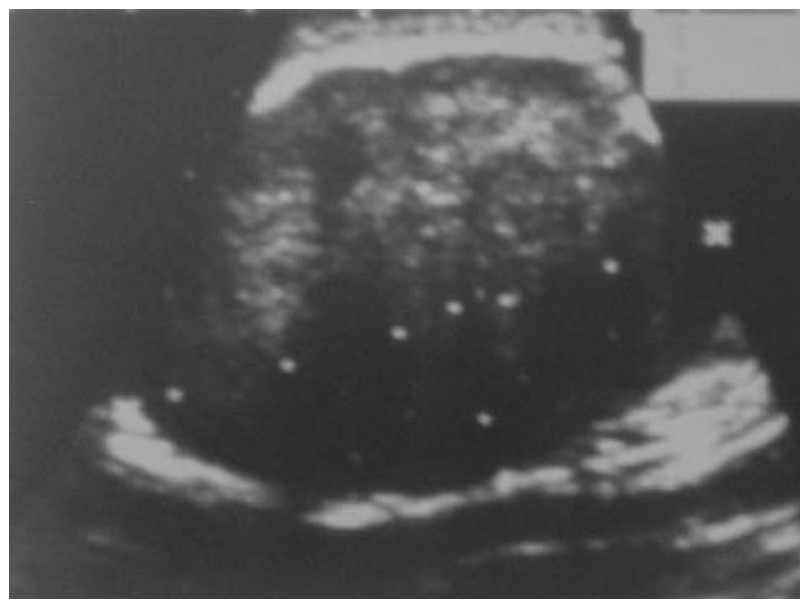

FIGURA 1. Patrón ecográfico hiperecogénico y heterogéneo.

El TAC abdómino-pélvico evidenció una masa homogénea escrotal izquierda que desplazaba al testículo homolateral y con planos respetados (Fig.2). Ausencia de adenopatías en todas las cadenas linfáticas estudiadas. No se detectó enfermedad a distancia.

Se completó el estudio radiológico mediante radiografia de torax que resultó normal.

Practicamos orquiectomía radical izquierda por vía inguinal alta con ligadura precoz de pedículo resultando el postoperatorio sin complicaciones.

La pieza quirúrgica mostró neoformación dependiente de cordón espermático de $7 \mathrm{~cm}$ de diámetro. Se trataba de un tumor pseudoencapsulado, blanquecino, multinodular, de consistencia caucho y textura trabeculada y uniforme a la sección, sin áreas de necrosis o hemorragia (Fig. 3).

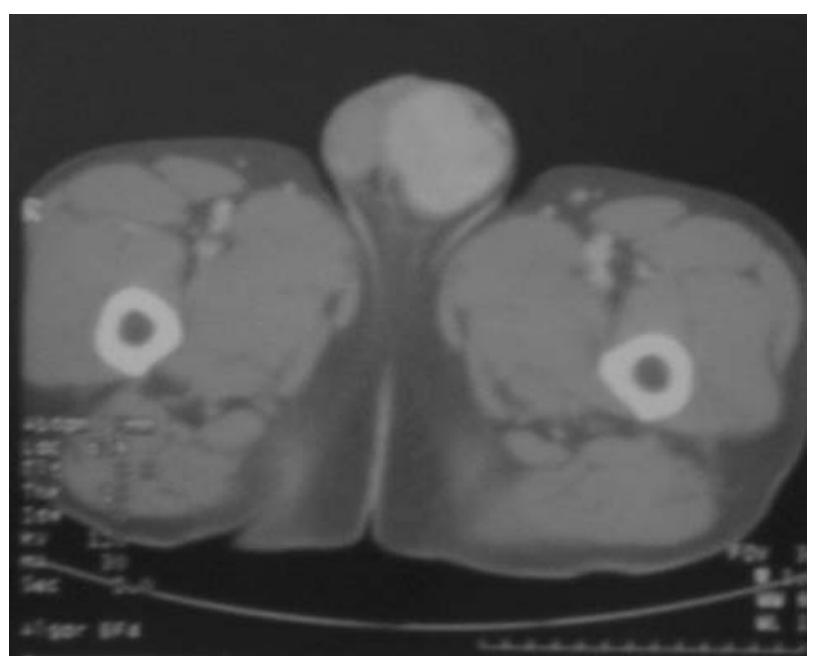

FIGURA 2. TAC abdómino-pélvico. Masa escrotal izquierda homogénea.

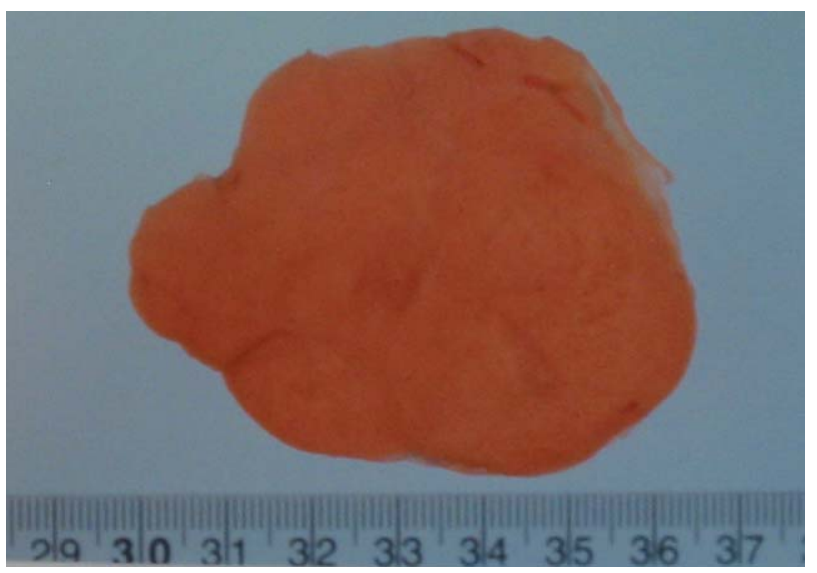

FIGURA 3. Tumor blanquecino, pseudoencapsulado y uniforme a la sección.

Histológicamente, la neoplasia se encontraba constituida por una proliferación homogénea de células fusiformes de hábito mesenquimal con disposición en fascículos confluentes en ángulo agudo (Fig. 4). Los núcleos fueron ovoides, regulares y se observó de 4 a 6 mitosis por 10 campos de gran aumento. La tinción de Gomori evidenció que las células aparecían rodeadas por fibras de reticulina (Fig. 5). El estudio inmunohistoquímico mostró positividad de las células tumorales para vimentina y negatividad para marcadores musculares como actina muscular y desmina. El índice de proliferación medido con Ki-67 resultó inferior al $1 \%$.

El diagnóstico fue de fibrosarcoma de bajo grado de malignidad y no se administró terapia postoperatoria alguna. Tras 5 años de segui- 


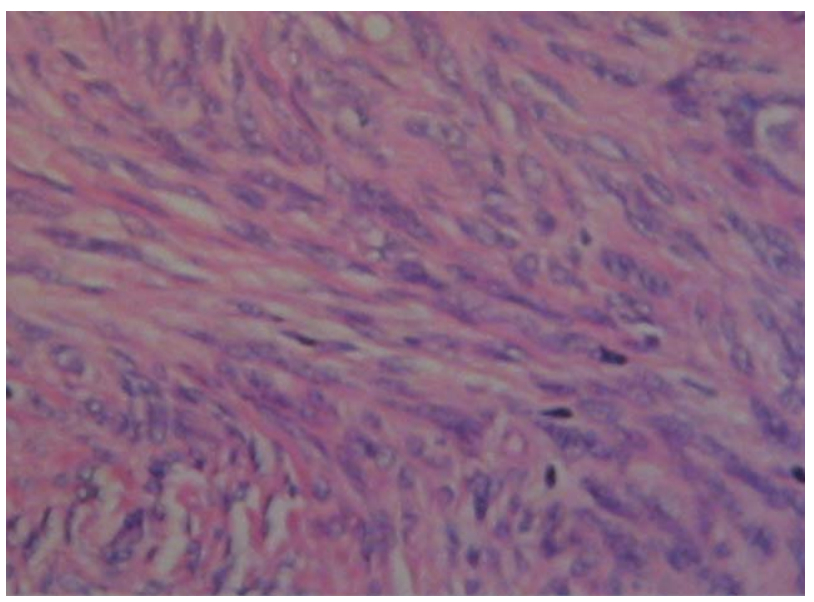

FIGURA 4. Hematoxilina-eosina 40x. Células fusiformes de hábito mesenquimal.

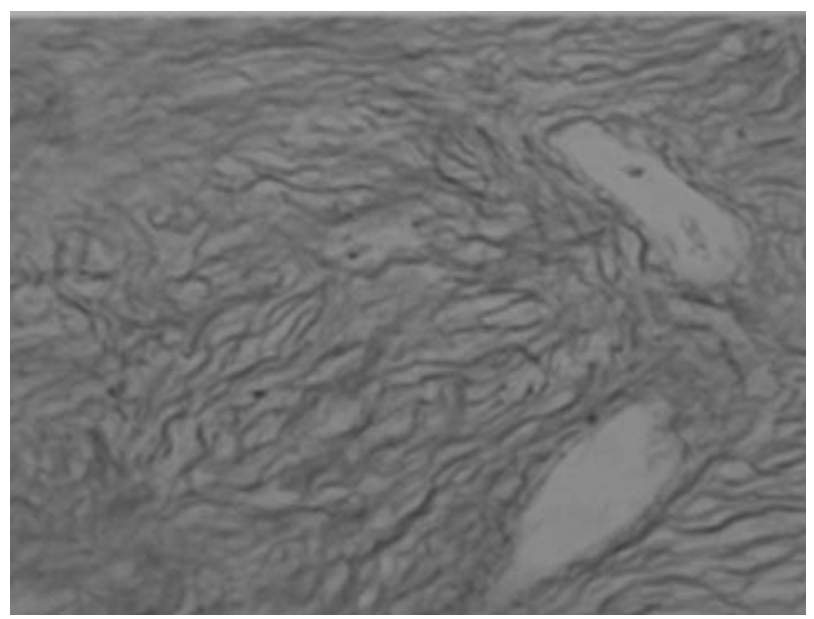

FIGURA 5. Tinción de Gomori 40x. Fibras de reticulina rodeando a las células.

miento, el paciente ha permanecido asintomático y sin signos clínicos ni radiológicos de recidiva local y/o a distancia.

\section{DISCUSIÓN}

Las neoplasias del cordón espermático son raras y representan sólo el $4 \%$ de los tumores escrotales $^{3}$. Las más frecuentes son las de estirpe benigna y de ellas los lipomas (45\%). Los sarcomas representan el $31 \%$ e incluyen rabdomiosarcomas, leiomiosarcomas, liposarcomas, histiocitoma fibroso maligno y fibrosarcomas. El rabdomiosarcoma en su forma juvenil, seguido del tumor desmoplásico de célula pequeña, también en niños, representan aproximadamente el $40 \%$ de todos los tumores paratesticulares tanto benignos como malignos. El leiomiosarcoma y el liposarcoma bien diferenciado son los más frecuentes en la edad adulta. El más infrecuente de todos ellos es el fibrosarcoma constituyendo sólo un $5 \%$ del total de estas neoplasias ${ }^{4-7}$.

El fibrosarcoma es un tumor de adultos, habitualmente, aunque puede aparecer en cualquier grupo de edad, incluso presentarse como una neoplasia congénita ${ }^{4}$.

Debido a que la mayoría de los tumores del cordón espermático aparecen en la zona inferior, se suelen expresar como masas intraescrotales, con lo que se plantea siempre el diagnóstico diferencial con los tumores testiculares primarios ${ }^{8}$. Ocasionalmente pueden debutar como escroto agudo por necrosis o hemorragia intratumoral.

Los sarcomas del cordón se cree que derivan de las células mesenquimatosas, que están atrapadas dentro del cordón espermático sin sufrir ninguna diferenciación ${ }^{2}$. El tejido mesenquimatoso mesoblástico sufriría un proceso degenerativo como consecuencia de un estímulo, siendo capaz de transformarse en diferentes tipos de sarcomas puros por diferenciación a tejido muscular, vascular o conectivo fibroso, como es el caso del fibrosarcoma, siempre que el proceso fuera monomórfico. La transformación polimórfica daría lugar a sarcomas mixtos. No obstante, hay autores $^{9}$ que plantean la teoría de la dediferenciación, de modo que a partir de un tejido diferenciado como es el caso concreto de un lipoma, se originaría mediante transformación un tumor maligno tipo liposarcoma.

El fibrosarcoma es un tumor de crecimiento lento y a diferencia del resto de sarcomas presenta escasa tendencia a metastatizar regionalmente y a distancia. Es excepcional la diseminación linfática $\mathrm{y} / \mathrm{o}$ hemática ${ }^{10}$, lo cual no obvia la realización necesaria de un estudio de extensión en todos los casos con TAC abdómino-pélvico y $\mathrm{Rx}$ de tórax. Se ha informado de su alta tendencia a la recidiva local $^{1,10}$, aunque esto no se corresponde con nuestra experiencia en este caso.

En contra de lo que admite la literatura ${ }^{1,10}$, nuestro caso ha tenido una presentación atípica mostrando un crecimiento rápido en tan sólo 2 meses. Esta forma de desarrollo no ha condicionado el pronóstico, puesto que no ha habido 
enfermedad metastásica ni recidiva local tras 5 años de seguimiento. Además el patrón histológico ha sido de bajo grado (indice de proliferación medido con Ki-67 menor del 1\%).

Desde el punto de vista diagnóstico, la ecografia es útil como método inicial de evaluación, por su rapidez, bajo coste, inocuidad y accesibilidad. Puede determinar el tamaño, localización, naturaleza quística o sólida de lesiones únicas o múltiples y su relación con estructuras vecinas; sin embargo, las características ecográficas de las diferentes masas de tejidos blandos no son específicas; varian considerablemente entre lesiones e incluso dentro de una misma lesión, existiendo un claro solapamiento entre lesiones benignas y malignas, por lo que no es posible establecer un diagnóstico preciso ${ }^{3}$.

El diagnóstico definitivo lo dará el estudio histopatológico una vez realizada la orquiectomía radical. La biopsia transescrotal está contraindicada por estar demostrada la fácil diseminación cuando se realiza dicha maniobra ${ }^{10}$.

Es de destacar, que en nuestro caso, el estudio inmunohistoquímico es positivo para vimentina, que es un marcador de tumor mesenquimatoso. Los marcadores para músculo como desmina y actina muscular resultaron negativos. Las fibras de reticulina que evidencia la tinción de Gomori, integran la sustancia fundamental configurada del tejido conectivo, en este tipo de sarcoma.

En cuanto al tratamiento existe unanimidad de criterio en lo que a orquiectomía por vía inguinoescrotal se refiere. Es imprescindible que el clampaje y la posterior ligadura de las estructuras vasculares y del cordón se lleve a cabo lo más alto posible, a nivel del anillo inguinal interno ${ }^{10}$. La linfadenectomía no está indicada.

En cuanto al empleo de quimio y/o radioterapia en el fibrosarcoma paratesticular es una terapéutica que no ha sido ampliamente documenta$\mathrm{da}^{11}$. A diferencia de lo que ocurre en otros sarcomas del cordón como los rabdomiosarcomas donde el empleo de quimioterapia se acepta de forma unánime, el tratamiento de elección en el fibrosarcoma por su comportamiento biológico es la orquiectomía radical y el seguimiento estricto para el despistaje de la recidiva local.

\section{REFERENCIAS}

1. Senovilla Pérez JL, Moreno Sierra J, Zazo Remojaro A, Resel Estevez L. Tumores Paratesticulares. Resel Estevez L, Moreno Sierra J, editors. Tratado de Oncología Urológica. Tomo II. Madrid, Sanidad y Ediciones S.L. 2003 pp 1037-1046.

2. Flores N, Unda M. Tumores Paratesticulares. Jiménez Cruz JF, Rioja Sanz LA, editors. Tratado de Urología. Tomo II. Barcelona, J.R. Prous Editores. 1993 pp1311-1322.

3. Rangel Villalobos E, Roldán Lora F, Canabal Berlanga A, Jimenez Lorente AI, Blanco Yun A. Escroto agudo postraumático como forma de presentación de rabdomiosarcoma embrionario paratesticular: diagnóstico ecográfico. Vox Paedriatica 2003; 11(2):37-40.

4. Arlen M, Grabstald H, Whithmore WF Jr. Malignant tumors of the spermatic cord. Cancer 1989,23(3):525-532.

5. Alexander F. Intrascrotal Sarcomas. Br J Cancer 1968;22 (3):486-497.

6. Blitzer PH, Dosoretz DE, Proppe KHG. Treatment of malignant tumors of the spermatic cord: a study of 10 cases and review of the literature. J Urology 1981;126:611-614.

7. Srigley JR. The paratesticular region: histoanatomic and general considerations. Seminars in Diagnostic Pathology. 2000; 17(4):258-269.

8. Algaba F, Moreno A, Trias I. Uropatología Tumoral: correlación morfológica, molecular y clínica. $1^{a}$ Edición. Barcelona: Pulso Ediciones S.A, 1996.

9. Peyrí Rey E, Urban Ramón A, Martinez Fernandez M, Sanmarti da Silva B. Liposarcoma rediferenciado del cordón espermático: degeneración de un lipoma previo resecado. Actas Urol Esp. 2003;27(5):383-386.

10. Castiñeiras J, Varo C, Sanchez Bernal A, Juarez A, Castro C, Lopez A et al. Neoplasias paratesticulares de origen mesenquimatoso. Estudio clínico-patológico. Actas Urol Esp. 1995; 19(1):40-45.

11. Calahorra Fernandez FJ, Perez Seoane C, Ramos A, Rodriguez Antolin A, Polo G, Leiva O. Liposarcoma paratesticular del cordón espermático. Actas Urol Esp. 1990: 14(3):202-204.

Dr. J.E. Blasco Alfonso

E-mail: karian@ono.com

(Trabajo recibido el 31 de agosto 2005) 